\title{
Transcript and protein expression decoupling reveals RNA binding proteins and miRNAs as potential modulators of human aging
}

\author{
Yu-Ning Wei ${ }^{1,2}$, Hai-Yang Hu ${ }^{1}$ Gang-Cai Xie ${ }^{1,2}$, Ning Fu ${ }^{3}$, Zhi-Bin Ning ${ }^{3}$, Rong Zeng ${ }^{3 *}$ and Philipp Khaitovich ${ }^{1,4,5^{*}}$
}

\begin{abstract}
Background: In studies of development and aging, the expression of many genes has been shown to undergo drastic changes at mRNA and protein levels. The connection between mRNA and protein expression level changes, as well as the role of posttranscriptional regulation in controlling expression level changes in postnatal development and aging, remains largely unexplored.

Results: Here, we survey mRNA and protein expression changes in the prefrontal cortex of humans and rhesus macaques over developmental and aging intervals of both species' lifespans. We find substantial decoupling of mRNA and protein expression levels in aging, but not in development. Genes showing increased mRNA/protein disparity in primate brain aging form expression patterns conserved between humans and macaques and are enriched in specific functions involving mammalian target of rapamycin (mTOR) signaling, mitochondrial function and neurodegeneration. Mechanistically, aging-dependent mRNA/protein expression decoupling could be linked to a specific set of RNA binding proteins and, to a lesser extent, to specific microRNAs.
\end{abstract}

Conclusions: Increased decoupling of mRNA and protein expression profiles observed in human and macaque brain aging results in specific co-expression profiles composed of genes with shared functions and shared regulatory signals linked to specific posttranscriptional regulators. Genes targeted and predicted to be targeted by the aging-dependent posttranscriptional regulation are associated with biological processes known to play important roles in aging and lifespan extension. These results indicate the potential importance of posttranscriptional regulation in modulating aging-dependent changes in humans and other species.

\section{Background}

Regulation of gene expression is a fundamental process controlling the implementation of genetic information. While a large part of expression regulation takes place in the transcriptional stage, posttranscriptional regulation also plays critical roles in controlling biological processes. Specifically, posttranscriptional regulation has been shown to contribute to the fine-tuning of gene expression in such cellular processes as apoptosis, immune response, inflammation, neuronal differentiation, synaptic plasticity, the cell cycle, and oncogenesis [1-3]. In all

\footnotetext{
*Correspondence: zr@sibcb.ac.cn; khaitovich@eva.mpg.de

${ }^{3}$ Key Laboratory of Systems Biology, Institute of Biochemistry and Cell Biology, Shanghai Institutes for Biological Sciences, Chinese Academy of Sciences, 320 Yue Yang Road, Shanghai 200031, China

${ }^{1}$ CAS Key Laboratory of Computational Biology, CAS-MPG Partner Institute for Computational Biology, 320 Yue Yang Road, Shanghai 200031, China
} Full list of author information is available at the end of the article these cases, posttranscriptional regulation was exerted by molecular crosstalk between cis-acting sequence elements located on the target RNA and trans-acting regulatory factors: RNA binding proteins (RBPs) and noncoding RNAs, such as microRNAs (miRNAs) [4,5].

The human genome encodes as many as 500 known and predicted RBPs identified based on the presence of RNA-binding domains [6]. It is known that RBPs exert their function through binding to specific sequence elements located predominantly but not exclusively in the 3' UTR of the transcript [7], such as AU-rich elements [8]. Exact binding specificity, however, has been studied for fewer than 50 human RBPs to date [6]. At present, most knowledge of RBP binding sites has been obtained through large-scale crosslinking and immunoprecipitation (CLIP) experiments, including high-throughput sequencing of RNA isolated by CLIP (HITS-CLIP) [9], 
photoactivatable-ribonucleoside-enhanced CLIP (PARCLIP) [10], and individual nucleotide resolution CLIP (iCLIP) [11]. Despite the relatively small number of studies focusing on RBPs, these proteins have been shown to play important roles in controlling splicing, polyadenylation, stability, editing, localization, and translational efficiency of RNA transcripts in human tissues and cell lines [5].

Human miRNAs comprise a large family of over 1,800 small noncoding RNAs with a length of 21 to 25 nucleotides [12]. Functionally, miRNAs guide RNA-induced silencing complex (RISC) to target transcripts in conjunction with Argonaut (AGO) RBPs [13]. Target recognition is mediated by partial binding of an miRNA sequence to a complementary region commonly located in the 3' UTR of a transcript [14]. While in some cases miRNAs have been shown to function as translational activators, in the vast majority of cases they act as posttranscriptional repressors, reducing the stability and/or translational efficiency of the target transcripts [15].

Expression of many genes has been shown to undergo drastic changes during human development and/or aging at both mRNA and protein levels [16]. Posttranscriptional regulation, especially that mediated by miRNAs, has been repeatedly implicated in the control of gene expression changes at specific developmental transitions in a number of species, including humans [17]. Still, the long-term effects of posttranscriptional regulation on organ and tissue development remain largely unexplored. In aging, regulation of mRNA translation has been shown to modulate longevity in a wide range of model organisms, from yeast to mice [18-21]. Despite this, the extent of posttranscriptional regulation in human development and aging and, specifically, its role in uncoupling protein and mRNA expression changes have not yet been investigated.

In this study, we surveyed transcriptome and proteome changes taking place during postnatal development and aging in humans and rhesus macaques, in a specific brain region, the prefrontal cortex (PFC). In both species, we observed substantial decoupling of mRNA and protein levels in aging, but not during the developmental interval. Genes showing increased mRNA/protein abundance decoupling formed expression patterns conserved between humans and macaques and were enriched in functions associated with lifespan regulation and senescence: mammalian target of rapamycin (mTOR) signaling, mitochondrial function and neurodegeneration. All mRNA/protein expression profiles found in human and macaque brain aging could be linked to specific posttranscriptional regulators, RBPs and miRNAs, based on binding site specificity data determined in large-scale CLIP-seq experiments.

\section{Results}

Age-dependent mRNA and protein expression in human and macaque brains

To determine the role of posttranscriptional regulation in development and aging, we assessed protein expression levels in human and macaque brains using timeseries data collected over the species' lifespans and compared these with mRNA expression levels measured in a largely overlapping set of samples from prior studies (Table S1 in Additional file 1).

We quantified mRNA expression levels based on highthroughput RNA sequencing (RNA-seq) data for the PFC of 14 humans and 15 rhesus monkeys [22]. These data span most of the species' lifespans: from 2 days to 98 years in humans and from 1 day to 28 years in macaques (Figure 1A; Tables S2 and S3 in Additional file 1). To quantify and compare gene expression in the two species in an unbiased manner, we mapped human and macaque RNA-seq reads to the consensus genome constructed based on pairwise genome alignment of human and rhesus macaque genomes. This resulted in 213 million (72.4\%) human and 323 million (65.7\%) macaque uniquely mapped reads. Based on these reads, 11,734 and 12,097 protein-coding genes were classified as expressed in human and macaque time series (Table S4 in Additional file 1).

We measured protein expression levels using labelfree quantitative mass spectrometry in PFC samples from 12 humans and 12 rhesus macaques with age distributions mirroring those of the transcriptome measurements: between 1 day and 98 years for humans, and between 16 days and 28 years for macaques (Figure 1A; Tables S5 and S6 in Additional file 1). Of the 12 human samples used for protein measurements, 10 were represented in the mRNA dataset (Tables S2, S3, S4 and S5 in Additional file 1). With the peptide identification false discovery rate (FDR) set to $1 \%$, we identified a total of 754,258 and 689,880 peptides corresponding to 8,011 and 7,708 genes in human and rhesus macaque samples, respectively. Of these genes, 2,278 and 2,351 were reliably detected in at least half of human or macaque samples (Table S7 in Additional file 1).

General analysis of mRNA and protein expression variation among human PFC samples indicated that age explains a substantial proportion of expression differences among samples in both datasets (Figure 1B,C; Figure $\mathrm{S} 1$ in Additional file 1). Consistently, of the 11,734 genes expressed in the human PFC, 6,955 showed significant expression changes with age $(P<0.05$ after Benjamini correction, Fisher's test). Of these, 1,963 (28.2\%) were also detected reliably at the protein level. For these genes, we compared protein and mRNA expression changes with age at 20 time points, interpolated using spline curves fitted to the actual expression data 


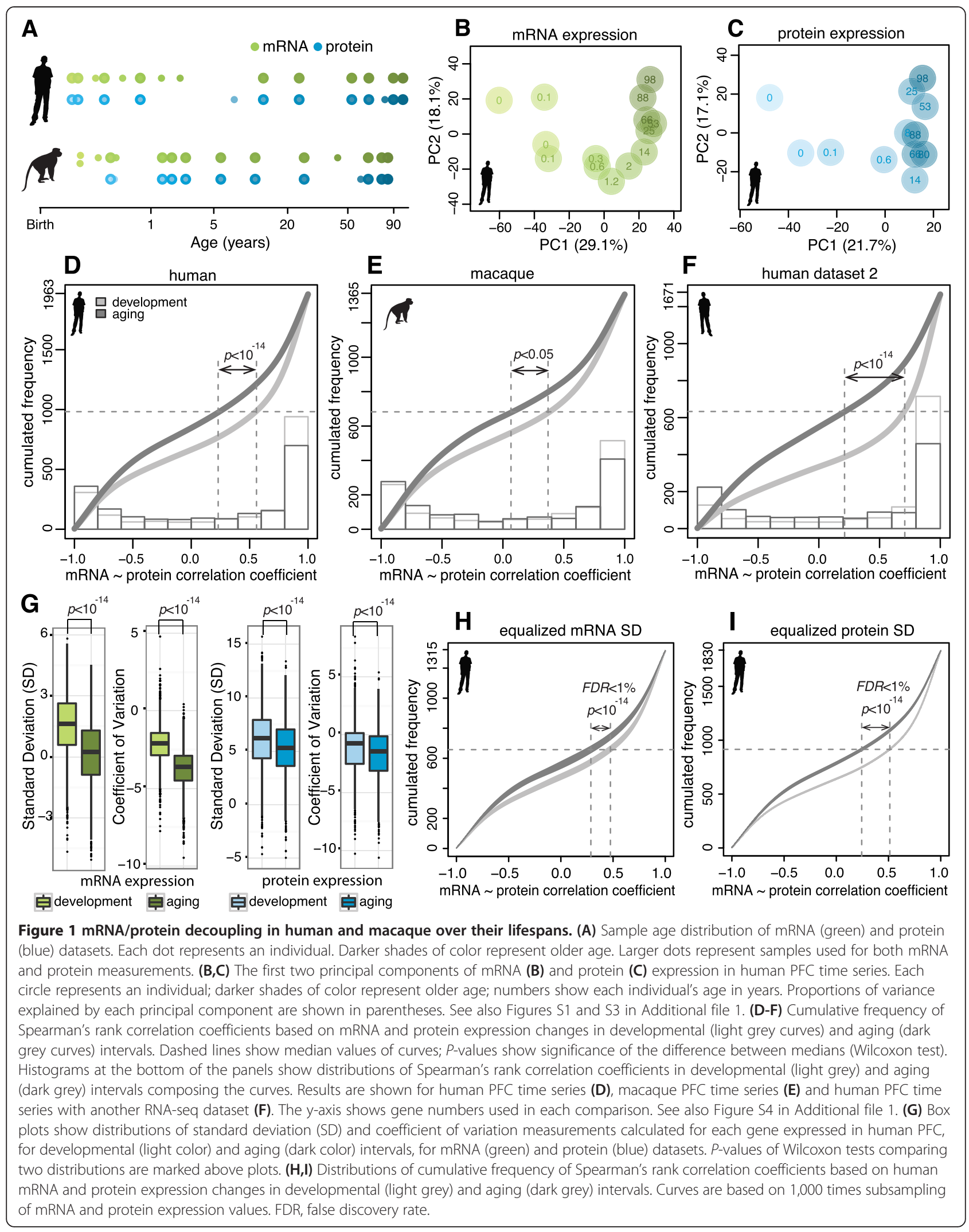


(Figure S2 in Additional file 1). Following other studies, we separated the developmental and aging intervals based on the age of sexual maturity [23,24], with 10 time points interpolated at each of these intervals. Similar procedures were applied to the rhesus macaque agedependent genes (Figure S3 in Additional file 1) [25,26].

\section{Increased decoupling of mRNA and protein expression in aging}

Substantial influence of posttranscriptional regulation on developmental or aging processes may result in detectable decoupling of mRNA and protein expression changes with age. To measure the concordance of mRNA and protein expression profiles, we used Spearman's rank correlation coefficients. Results of the correlation analysis differed drastically between the developmental and aging lifespan intervals: the concordance of mRNA and protein expression levels was much higher during development than during aging $(P<0.001$, Wilcoxon test; Figure 1D-F). The increased decoupling of mRNA and protein expression levels in aging was significant for both the decrease of positive correlations and the increase of negative correlations $(P<0.05$, FDR $<5 \%$, Spearman's rank correlation) in the aging interval $(P<0.05$, chi-square test; Figure S4A in Additional file 1).

Increased decoupling of mRNA and protein expression levels in aging was not caused by higher expression variation among human individuals of older age. It was also not caused by a difference in the expression levels or by the amplitude of expression changes between the developmental and aging intervals. Specifically, in our dataset, mRNA and protein expression variation was significantly smaller in the aging than the developmental interval $(P<0.001$, Wilcoxon test; Figure 1G). Further, subsampling of data using equalized distribution of standard deviation, coefficient of variation, expression levels or expression change amplitudes for developmental and aging intervals did not affect our results (Figure 1H,I; Figure S4B-D in Additional file 1).

The increased decoupling of mRNA and protein expression levels during aging could be reproduced in the rhesus macaque dataset. Firstly, repeating our analysis based on macaque mRNA and protein time series data, we observed a similar increase in the discordance of mRNA and protein expression changes in the aging interval (Figure 1E; Figure S4E in Additional file 1). Secondly, genes showing concordant and discordant mRNA/protein expression in human aging overlapped significantly with genes showing concordant and discordant expression in macaque aging $(P<0.005$, permutations) (Figure 2A,B; Figure S5A in Additional file 1). Genes showing concordant and discordant mRNA/ protein expression in aging were defined based on a significant positive correlation in the developmental interval and significant positive (concordant genes) or negative (discordant genes) correlations in aging $(P<0.05$, FDR $<5 \%$, Spearman's rank correlation). In humans, 359 genes were classified as concordant and 260 as discordant (Figure 2A). The fact that increased mRNA-protein disparity could be reproduced in the macaque time series is noteworthy, as the macaque samples were collected from individuals kept in the same standard living conditions and were not subjected to biological and technical artifacts associated with differences in agonal state and post mortem delay.

In addition to the macaque data, increased decoupling of mRNA and protein expression levels during aging could be reproduced using a different published human RNA-seq time series dataset containing data from 38 individuals [27] (Figure 1F; Table S8 in Additional file 1). Comparing human mRNA expression profiles with macaque protein expression profiles and vice versa reproducibly showed greater mRNA/protein expression decoupling in aging (Figure S4F,G in Additional file 1). Furthermore, the concordant and discordant gene groups defined based on any of the above-mentioned comparisons overlapped significantly with corresponding gene groups defined based on the original human dataset $(P<0.005$, permutations; Figure S5B-G in Additional file 1). Thus, increased decoupling of mRNA and protein expression levels during human and macaque brain aging can be reproducibly observed in multiple datasets and are not likely to be caused by technical artifacts or environmental differences between developmental and aging intervals.

To assess whether increased decoupling of mRNA and protein expression levels in brain aging could be caused by use of different individuals for mRNA and protein measurements or differences in individuals' ethnicity, we repeated all analyses based on a set of 10 human individuals for which both mRNA and protein data were generated, and based on a subset of 9 of these 10 individuals (all nine being of Caucasian descent). The results of these analyses were in full concordance with those obtained using the full dataset (Figures S6 and S7 in Additional file 1).

\section{Concordant and discordant genes form conserved co-expressed clusters}

To investigate possible functional implications of increased mRNA/protein expression decoupling in human aging, we sorted 359 concordant and 260 discordant genes into co-expressed clusters based on their mRNA expression patterns over the lifespan. A non-supervised hierarchical clustering revealed four main patterns: (P1) mRNA expression continues to decrease across both lifespan intervals; (P2) a decrease in development followed by stabilization or increase in aging; (P3) an 


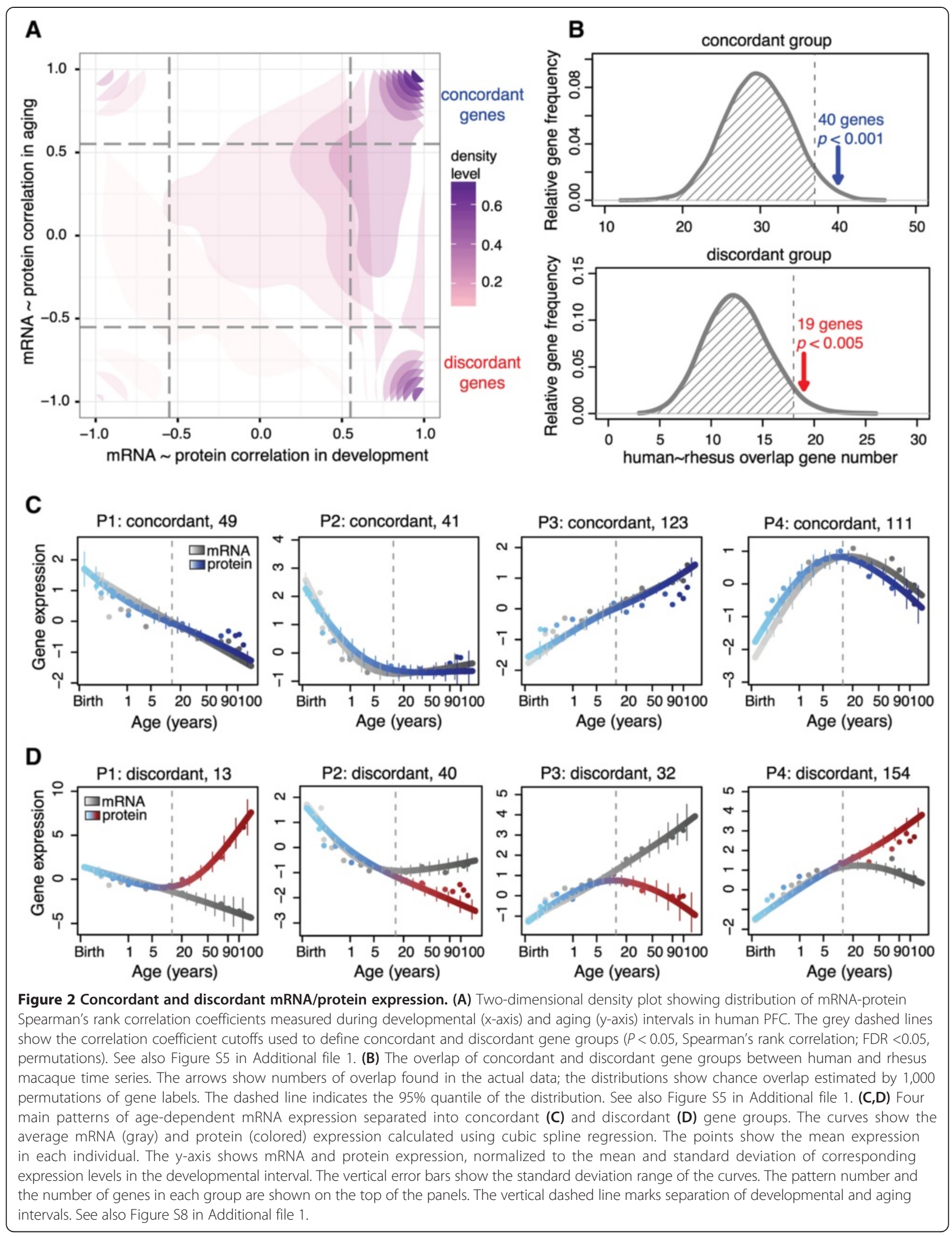


increase that continues across the lifespan; and (P4) an increase in development followed by stabilization or a decrease in aging. Within each mRNA co-expression pattern, we then separated the concordant and discordant gene groups (Figure 2C,D). The numbers of genes constituting these gene groups within each pattern varied widely from 11 to 154 . Still, for all eight gene groups, the average expression trajectories found in the human data could be reproduced in rhesus macaque time series (Figure S8 in Additional file 1). Thus, the obtained concordant and discordant expression profiles are largely conserved between human and macaque brain development and aging.

Conservation of protein and mRNA co-expression profiles, and their concordant and discordant relationships, suggests this phenomenon is functionally important. Indeed, all eight gene groups show significant enrichment in specific functional terms and pathways specified by Gene Ontology (GO) and Kyoto Encyclopedia of Genes and Genomes (KEGG) annotations [28,29]. While concordant genes are mainly enriched in signal transduction, nucleotide binding and ATP binding, discordant genes show a tendency towards regulatory and signaling functions. Thus, P1 discordant genes are enriched among transcriptional regulators, including zinc finger proteins, and P2 ones in protein binding, kinase activity and translation. Discordant genes in both P2 and P3 are overrepresented in the insulin receptor signaling pathway and mTOR signaling pathway. P4 discordant genes are enriched in mitochondrion, ATP biosynthetic process, calcium ion binding, Huntington's disease and Parkinson's disease (Additional file 2).

Specific RNA binding proteins mediate mRNA and protein expression decoupling in brain aging

Increased decoupling of mRNA and protein expression levels in human brain aging could potentially be caused by actions of common posttranscriptional regulators RBPs and miRNAs $[4,5]$.

To assess the role of RBPs in increased mRNA/protein disparity, we compiled human transcriptome-wide maps of endogenous binding sites for 17 RBPs from 13 families based on published large-scale CLIP experiments, including HITS-CLIP, PAR-CLIP and iCLIP experimental data [11,30-37] (Table S10 in Additional file 1). To focus on posttranscriptional regulatory effects corresponding to expression level differences between mature mRNAs and proteins, we excluded binding sites located in intronic regions or splice junctions. For each expression pattern, we compared the distributions of RBP binding sites in concordant and discordant gene groups, which differ only by protein expression trajectories in the aging interval. Thus, within the same pattern, differences in RBP binding site distribution should reflect differences in posttranslational regulation between concordant and discordant groups during aging.

In agreement with our predictions, we found significant enrichment of RBP binding site number and density in discordant gene groups, compared with the concordant groups, for all four expression patterns (Figure 3). Within each discordant group, binding sites corresponding to enriched RBPs covered the majority of genes. Furthermore, the expression patterns of enriched RBPs showed specific correlations with their putative target genes in the corresponding pattern. By contrast, reciprocal analysis of concordant groups yielded no such enrichment of common RBP regulators (Figure S9 in Additional file 1).

Specifically, discordant genes within the P1 pattern show significant enrichment of SFRS1 binding sites $(P<0.05$, Wilcoxon test after Bonferroni correction). Notably, among 13 genes in this group, 10 (77\%) contained at least one SFRS1 binding site. Furthermore, SFRS1 expression significantly correlated positively with protein expression, but not mRNA expression, for 10 putative target genes $(P<0.01$, Spearman's rank correlation; Figure 3E; Figure S10 in Additional file 1). This positive relationship is consistent with previous reports demonstrating the role of SFRS1 as a translational activator in HeLa cells both in vivo and in vitro [38].

Similarly, discordant genes within the P2 pattern show significant enrichment of TIAL1 binding sites: as many as $34(85 \%)$ of the 40 genes in this group contain at least one TIAL1 binding site $(P<0.05$, Wilcoxon test after Bonferroni correction). In aging, TIAL1 expression correlated significantly and negatively with protein expression and significantly and positively with mRNA expression for the 34 putative target genes $(P<0.01$, Spearman's rank correlation; Figure 3F). Again, this regulatory relationship is consistent with studies reporting that TIAL1 functions as a translational repressor exhibiting no detectable inhibitory effect at the mRNA level [39].

Genes within the P4 pattern form the largest group, with 154 co-expressed discordant genes. Genes within this group show significant enrichment for seven RBPs: TAF15, MOV10, AGO2, SFRS1, IGF2BP2, TIA1 and TIAL1 $(P<0.05$, Wilcoxon test after Bonferroni correction). All 154 genes in this group contain at least one binding site for one of the seven enriched RBPs, suggesting that the RBPs examined in our study are, in principle, sufficient to explain the discordant behavior for all genes within this group. With the exception of TAF15, expression profiles of the enriched RBPs correlated positively with the protein expression profiles of their putative target genes in the aging interval (Figure 3H). 


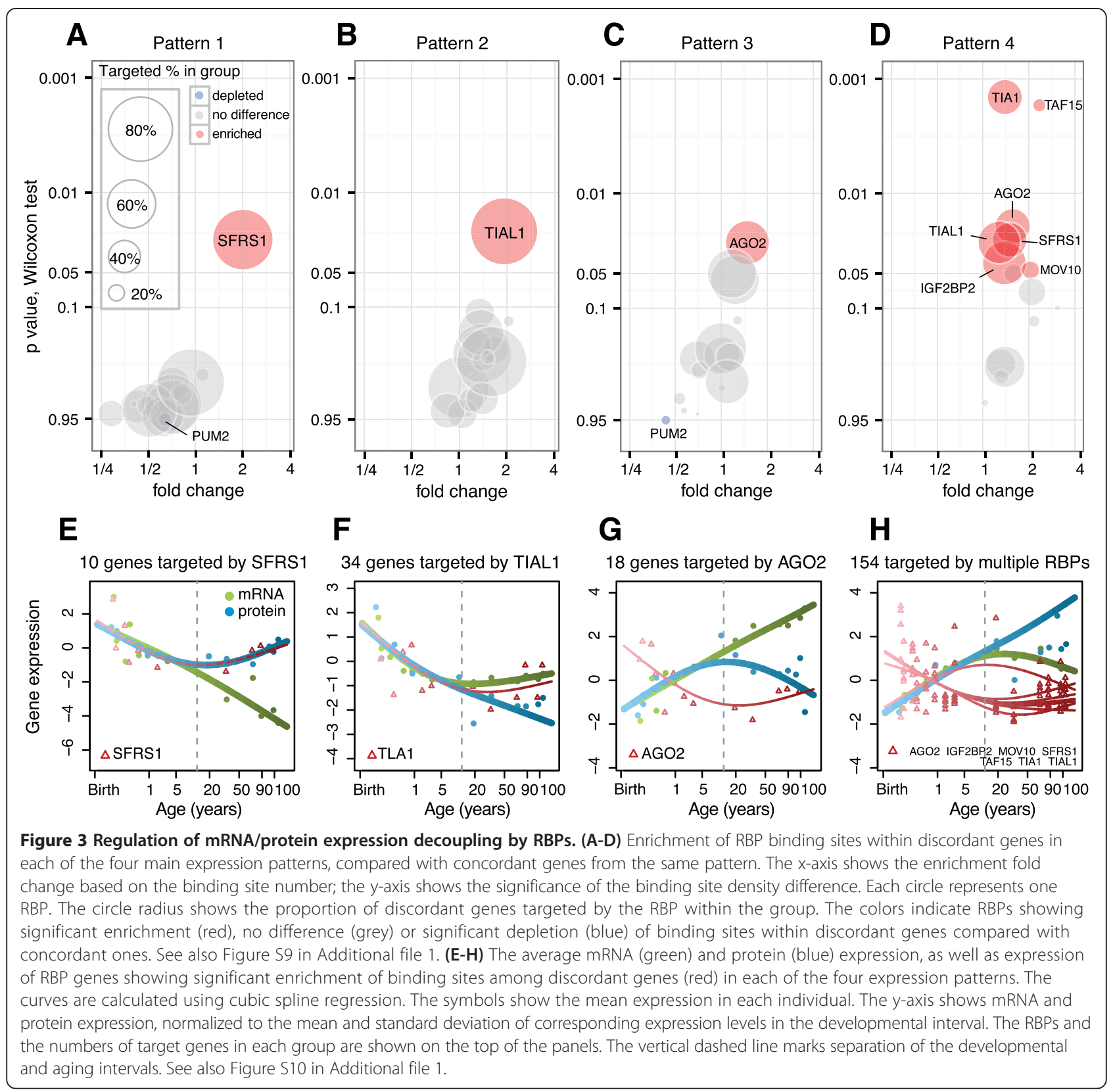

Role of miRNAs in mRNA and protein expression decoupling While decoupling between mRNA and protein expression profiles in human brain aging may, in principle, be driven by RBPs in the P1, P2 and P4 patterns, discordant genes within the P3 pattern show a diverse set of regulatory characteristics. The only RBP showing significant binding site enrichment in the P3 discordant group is AGO2, with 18 (56\%) of the 32 genes in this group containing AGO2 binding sites. The number of AGO2 binding sites per gene was nonetheless greater in this group than in the other three discordant groups (Figure 4A). Functionally, AGO2 mediates posttranscriptional regulation as one of the main components of RISC [13]. In agreement with the inhibitory role played by RISC, we see a significant negative correlation between AGO2 expression and protein expression of its putative target genes in the P3 discordant group $(P<0.01$, Spearman's rank correlation; Figure $3 G)$. Specificity of the RISC inhibition, however, is mainly determined by another component of the complex - miRNA [14].

To appraise the possible effects of miRNAs on decoupling mRNA and protein expression profiles in the P3 and other discordant groups, we analyzed published miRNA data collected from the same 12 human individuals used in our proteome analysis [40] (Table S5 in Additional file 1). Of the 373 miRNAs we reliably 


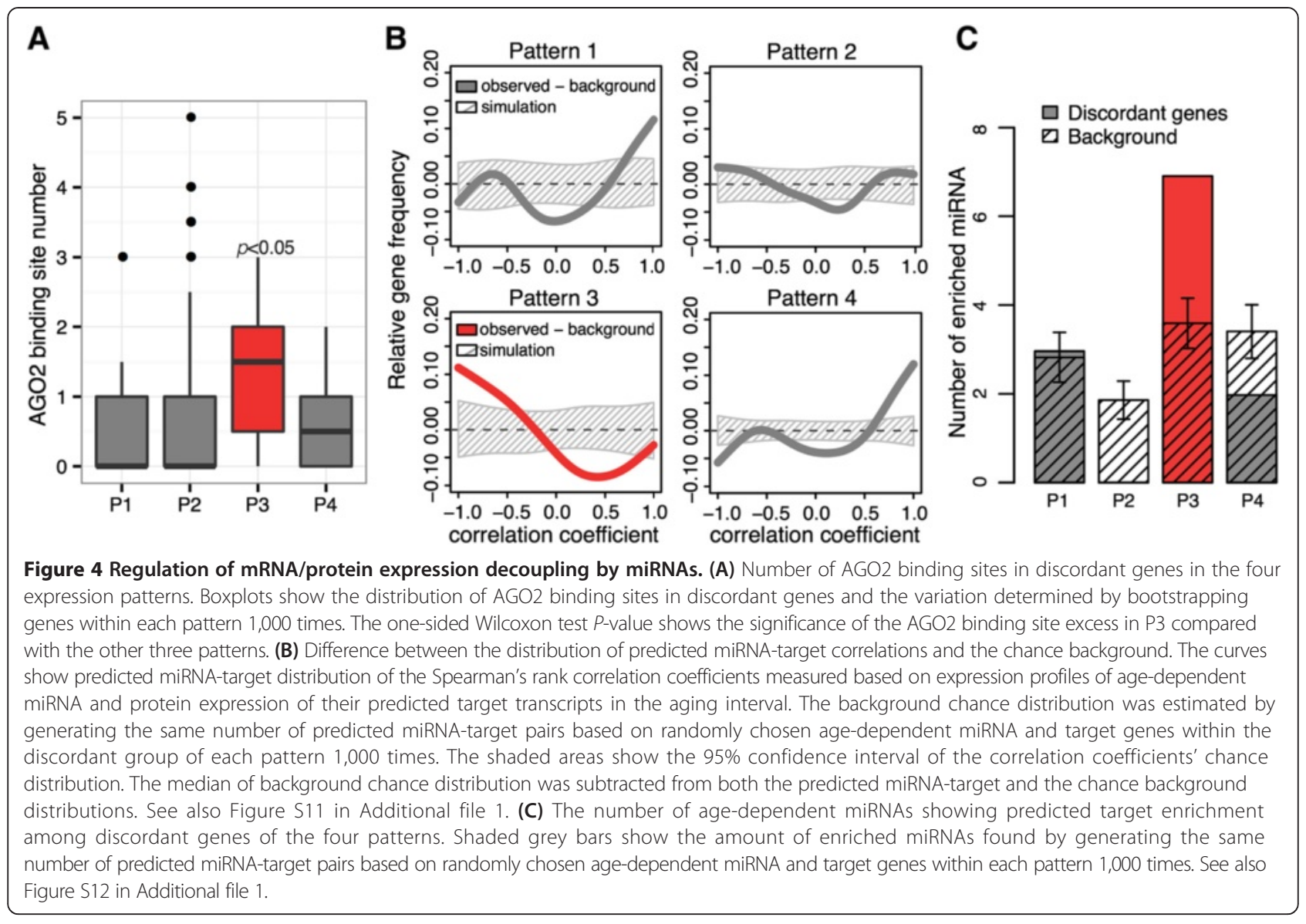

detected in the dataset, 201 showed significant agedependent expression level changes $(P<0.05$, Fisher's test after Benjamini correction). For these miRNAs, we predicted the locations of conserved target sites, and assessed the correlation between miRNA expression and expression of their predicted target genes in the four discordant groups. Only the P3 discordant group displayed a significant negative correlation between miRNA expression and protein expression of predicted targeted genes in the aging interval $(P<0.05$ by simulation; Figure $4 \mathrm{~B})$. This result was robust to the choice of miRNA target prediction tools using various prediction strategies [41,42] (Figure S11 in Additional file 1). Concordantly, only the P3 discordant group contained a greater than expected number of binding sites for specific miRNAs: out of 12 age-dependent miRNAs showing significant binding site enrichment in any of the four discordant groups, seven were associated with the P3 pattern $(P<0.05$, hypergeometric test after Bonferroni correction) (Figure 4C; Figure S12 in Additional file 1; Table S11 in Additional file 1). Taken together, these observations suggest that, in contrast to the P1, P2 and P4 expression patterns (where the decoupling of mRNA and protein expression levels in human aging might largely be driven by RPBs alone), mRNA/protein expression decoupling in the P3 pattern may result from cooperative regulation by AGO2 and miRNAs.

\section{Discussion}

In this study, we observed substantial decoupling of mRNA and protein expression profiles during human and rhesus macaque brain aging, but not in the developmental ontogenetic interval. Our results further indicate that all four patterns of aging-dependent mRNA/protein expression decoupling can be associated with a small number of key regulatory RBPs, such as SFRS1, TIAL1 and AGO2. It must be noted that our analyses were limited to experimentally defined RBP targets, mainly identified in cell line experiments. Consequently, many regulatory interactions could have been missed by our analyses. Nonetheless, our results maintain that agedependent decoupling of mRNA and protein expression patterns could be linked to specific posttranscriptional regulators.

In contrast to the RBP regulatory signal, only one of the four mRNA/protein decoupling patterns characteristic of primate brain aging showed a detectable signature of miRNA-mediated posttranscriptional regulation. This 
result indicates that the role of RBP-driven regulation of age-dependent changes may be underappreciated at this time.

We observed substantially higher discordance of mRNA and protein expression profiles in aging than in the developmental interval in both humans and macaques. Conservation of age-dependent decoupling profiles between humans and macaques, enrichment of genes following the same decoupling profiles in specific biological processes, as well as association of coexpressed gene groups with specific RBPs and miRNAs, suggest potential functionality of observed posttranscriptional regulation.

One of the strongest signals of RBP-mediated regulation of mRNA/protein discordant profiles that we detected in primate brain aging was TIAL1 association with P2 discordant genes. TIAL1 has been shown to be involved in various forms of translational control [39], including regulation of translation initiation pathways such as the mTOR pathway $[43,44]$. Notably, P2 discordant genes are significantly enriched in the mTOR pathway $(P<0.005$, hypergeometric test after Benjamini correction). Conversely, mTOR pathway genes are significantly overrepresented among genes showing discordant expression profiles in primate brain aging $(P<0.01$, chi-square test). This finding is especially noteworthy, as the mTOR pathway represents one of the few pathways shown to be involved in longevity regulation in a wide range of species [45-48].

Within the mTOR pathway, all six genes showing discordant expression patterns concentrate within the phosphoinositide 3-kinase (PI3K)-Akt-mTORC1 signaling cascade (Figure 5). This cascade is one of the central, evolutionarily conserved regulators of species' longevity $[49,50]$. The best-known function of mTORC1 signaling is the promotion of translation in the presence of extracellular stimuli, such as insulin, hormones and growth factors [51,52]. Extensive studies have shown that reducing activation of the PI3K-Akt signal-inhibiting mTORC1 activity could extend lifespan across multiple species, including mammals $[21,53,54]$. Moreover, aberrant control of the PI3K-Akt regulatory axis or overactivation of mTOR has been suggested to play a causal role in many aging-related disorders, including cancer, type 2 diabetes mellitus, heart disease and neurodegeneration $[50,55,56]$. Our study, as well as studies conducted in rats and mice, shows that $m T O R$ expression tends to increase with age $[57,58]$. On the other hand, mTOR activity was shown to decrease with age in the mouse hippocampus due to decreased activity of upstream signaling by PI3K-Akt [58].

Notably, all six genes showing discordant expression patterns within the PI3K-Akt-mTORC1 signaling cascade PI3K, Akt, mLST8, S6K1, eIF3e and eIF3f - were shown to

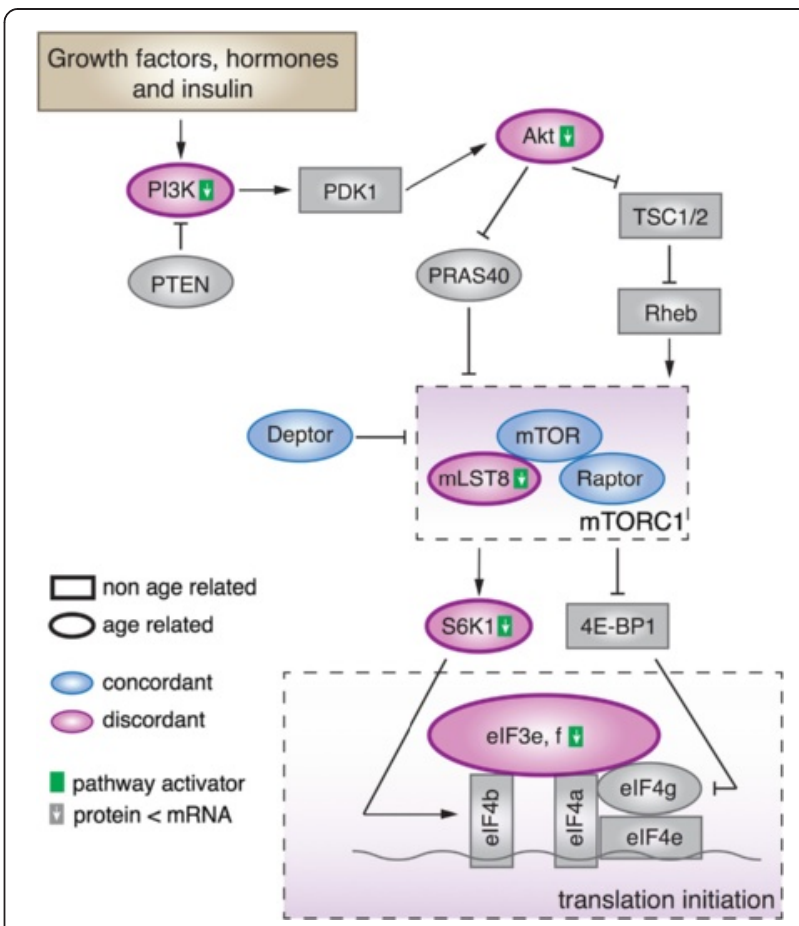

Figure 5 Discordant and concordant gene expression in the PI3K-Akt-mTORC1 signaling cascade. A schematic representation of selected components of the PI3K-Akt-mTORC1 signaling cascade based on [59-62]. The shapes indicate age-dependent (ovals) and non-age-dependent (rectangles) expression of pathway components. The colors indicate concordant (blue) and discordant (rose) expression in the aging interval. For each discordant gene, the green square next to the gene name illustrates its activator function in the pathway, while the white arrow in the square indicates the mRNA-protein expression relationship detected in our data. See also Figure S13 in Additional file 1.

function as mTOR pathway activators [59-62]. Furthermore, in our data, all of these genes showed the same discordant pattern - translational inhibition in primate brain aging (Figure S13 in Additional file 1).

Of genes showing discordant expression patterns within the PI3K-Akt-mTORC1 signaling cascade, several were directly implicated in lifespan regulation. For example, inhibition of PI3K and Akt or mutations in S6K1 have been shown to extend lifespan in yeast, nematodes, fruit flies and mice [21,53,54,63-67]. Similarly, mutation or knockdown of eIFs (translation initiation factors) have been shown to extend lifespan in yeast and nematodes $[19,68]$.

It has been proposed that a global reduction of mRNA translation could promote healthy aging, potentially by allowing endogenous protein repair and degradation machinery to maintain protein homeostasis in the face of protein damage and aggregation [69]. Furthermore, a general reduction in mRNA translation has been suggested to attenuate aging-related pathologies resulting from misplaced activity of biosynthetic and proliferative 
processes that are important in development but detrimental in aging [70]. Supporting this notion, the PI3KAkt-mTORC1 pathway has been validated as a potential target of cancer treatment [71]. Notably, the cancerprevention effect was achieved by repression of the PI3K-Akt-mTORC1 activity through TIAL1-mediated translational suppression [72]. Thus, the posttranscriptional regulation signals detected within the PI3K-AktmTORC1 pathway in our study may reflect an adaptive organismal response to increased accumulation of molecular damage in advanced age.

Another effect of mTORC1 dysfunction shown in a variety of species, from yeast to mice, is translational activation of mitochondrial genes and enhancement of mitochondrial respiration $[46,73,74]$. P4 discordant pattern-containing genes showing translational activation in primate brain aging are significantly enriched in mitochondria: as many as 37 of the 154 P4 discordant genes are nuclear-encoded mitochondrial proteins $(P<0.05$, hypergeometric test after Benjamini correction; Additional file 2). Among others, these genes include the mitochondrial translation initiator $m t I F 2$, the mitochondrial translation elongation factor $m t E F-T u$ and six components of the mitochondrial respiration chain. Reduction of bioenergetic efficiency and respiration efficacy mediated by mitochondria was shown to be one of the hallmarks of aging conserved across species [75,76]. Our data, showing translational activation of mitochondrial genes in primate brain aging, suggest that posttranscriptional regulation may play a role in augmenting aging-related decline of mitochondrial functionality. Supporting this notion, translational activation of mitochondrial genes mediated by mTORC1 inhibition has been shown to extend lifespan in yeast and fruit flies [77-79].

The P3 discordant group contains another example of posttranscriptional regulatory signaling, potentially linked to aging-related physiological changes taking place in the primate brain. Enrichment of AGO2 binding sites, combined with a significant negative relationship between miRNA expression and expression of their predicted target proteins, indicates that P3 discordant genes might be subject to miRNA-mediated translational repression. Notably, miRNAs linked with P3 discordant genes overlapped significantly with miRNAs showing aberrant expression in the prefrontal cortex of late-onset Alzheimer's disease patients $(P<0.001$, chi-square test $)$ [80]. Furthermore, one of the overlapping miRNAs, miR-132-3p, has been suggested to contribute to Alzheimer's disease progression through aberrant regulation $[81,82]$. Interestingly, mir-132-3p has been shown to be regulated by insulin signaling through the PI3KAkt-mTORC1 cascade [83]. Expression of some miRNAs linked with P3 discordant genes is increased in healthy aging, resulting in posttranscriptional inhibition of predicted P3 target genes (Figure S12 in Additional file 1). Interestingly, in Alzheimer's disease patients, expression of these miRNAs is decreased compared with healthy aging [80]. This suggests that impaired miRNAmediated posttranscriptional inhibition of P3 genes might be one of the important features of Alzheimer's disease.

\section{Conclusions}

Our results indicate that increased decoupling of mRNA and protein expression profiles reproducibly detected in human and macaque brain aging can be linked to specific posttranscriptional regulators - RBPs and miRNAs. Genes targeted and predicted to be targeted by the aging-dependent posttranscriptional regulation can be associated with biological processes known to play important roles in aging and lifespan extension, such as mTOR pathway, mitochondrial function and Alzheimer's disease. The directions of aging-dependent expression changes observed at the protein level further suggest the potential role of posttranscriptional regulation in counteracting the effects of aging decline. Taken together, these results indicate the potential importance of RBPmediated posttranscriptional regulation in controlling progression of the human aging phenotype.

\section{Materials and methods}

\section{Ethics statement}

Informed consent for the use of human tissues for research was obtained in writing from all donors or their next of kin. All macaques used in this study suffered sudden deaths for reasons other than their participation in this study and without any relation to the tissue used. The Biomedical Research Ethics Committee of Shanghai Institutes for Biological Sciences reviewed and approved the use and care of the animals in this research (approval ID: ER-SIBS-260802P).

\section{Sample collection}

We collected superior frontal gyrus samples from post-mortem brains of healthy humans and macaques (Tables S5 and S6 in Additional file 1; for details, see supplemental experimental procedures in Additional file 1).

\section{Protein sample preparation and label-free two-dimensional tandem mass spectrometry}

We followed the procedure of protein sample preparation and two-dimensional liquid chromatography coupled with tandem mass spectrometry (LC-MS/MS) analysis described in [84] in a pH continuous online gradient (pCOG) system. Briefly, proteins were extracted from $100 \mathrm{mg}$ of frozen prefrontal cortex tissue from 12 humans and 12 macaques (Tables S5 and S6 in Additional file 1). We extracted the protein samples and incubated them overnight with trypsin, followed by ultrafiltration and lyophilization. 
Lyophilized protein samples were then loaded on ion exchange columns and eluted using a $\mathrm{pH}$ continuous gradient buffer for the LC-MS/MS analysis (for details, see supplemental experimental procedures in Additional file 1).

\section{Pre-processing of RNA deep sequencing, miRNA deep sequencing and quantitative proteomics data} Human-macaque consensus reference genome construction Chained and netted alignment files for human (hg19) and macaque (rheMac3), aligned using BLASTZ [85], were downloaded from the UCSC Genome Browser. We used the human genome as a reference for all discordant genomic sites, and replaced insertions and deletions with 'N's in the consensus genome. Furthermore, 6 bp regions flanking each insertion or deletion site were masked by 'N's in the resulting human-macaque consensus reference genome (for details, see supplemental experimental procedures in Additional file 1).

\section{Computational pre-processing of mRNA deep sequencing}

The deep sequencing data of human and rhesus superior frontal gyrus of the PFC were obtained from the Sequence Reads Archive [86] under the accessions SRP005169 for [22] (Tables S1, S2 and S3 in Additional file 1).

To quantify and compare gene expression in the two species in an unbiased manner, we mapped human and rhesus RNA-seq reads to the human-macaque consensus reference genome, using STAR (v.2.3.0e) [87]. Potential PCR duplicates were removed, and only uniquely mapped reads were used in further analyses [88].

Gene expression was quantified as the number of reads per kilobase per million of total mapped reads (RPKM) by GENCODE annotation (v.17) [89,90].

Only genes with RPKM $\geq 1$ in more than two-thirds of the samples in one species (human or macaque) were classified as reliably expressed in that species and were used in the following analysis (for details, see supplemental experimental procedures in Additional file 1).

\section{Computational pre-processing of quantitative proteomics}

Peptides were identified by searching spectrums against the UniProt Knowledgebase (UniProKB) complete proteome human set [91], using the database search engine MS-GF+ [92]. We estimated the peptide-spectrum matches (PSM) level FDR of peptide identification by searching the combined database of the target dataset and the reversed decoy database. Only peptides with FDR $<1 \%$ were considered. Ten MS/MS scans of each sample were combined together.

Peptide data were mapped per gene to Ensembl genes by UniProtKB/Swiss-Prot and UniProtKB/TrEMBL annotations. Criteria for protein identification included detection of at least two unique peptides. Ambiguous peptides and redundant proteins were removed.
Quantification of protein expression of each gene was achieved by the normalized spectral abundance factor (NSAF) [93]. The counts of MS/MS spectra assigned to a protein were normalized to the length of the protein, resulting in a spectral abundance factor (SAF). Each SAF was further normalized against the sum of all SAFs in one sample, resulting in the NSAF value.

Only genes with a mean NSAF $\geq 1$ and a positive NSAF value in at least 6 of the 12 individuals were classified as reliably detected in a species (human or macaque), and were included in the downstream analysis (for details, see supplemental experimental procedures in Additional file 1).

\section{Computational pre-processing of miRNA deep sequencing}

The miRNA deep sequencing data for human PFC were obtained from the NCBI Gene Expression Omnibus [94] under series accession number GSE18069 for [40] (Tables S1, S2, S3, S4 and S5 in Additional file 1).

We followed the procedures of small RNA sequencing data pre-processing and miRNA expression quantification from [95]. All unique sequences were trimmed to remove the adapter sequence at the $3^{\prime}$ end. Trimmed sequences were mapped to the human genome (hg19) by the Bowtie algorithm [96], requiring a perfect match. We quantified miRNA expression by miRBase version 17 [97] with all perfect mapped sequences. The expression level of each miRNA was calculated as transcripts per million reads (TPM; the number of reads mapped to the transcript normalized by the number of total mapped reads; for details, see supplemental experimental procedures in Additional file 1).

\section{Computational pre-processing of age-dependent genes}

We followed the steps of [98] to test the effect of age on mRNA expression level, using polynomial regression models. We used the power of 0.25 of donor age (for details, see supplemental experimental procedures in Additional file 1) to simultaneously capture developmentaland aging-dependent changes. For each detected gene, we chose the best regression model with scaled age as predictor and mRNA expression level as response, using families of polynomial regression models and the 'adjusted $\mathrm{r}^{21}$ criterion. The significance of the chosen regression model was estimated using the F-test, and BenjaminiHochberg correction was carried out for all tested genes as multiple test correction (for details, see supplemental experimental procedures in Additional file 1).

\section{Analysis of mRNA-protein disparity}

To analyze the correlation between transcriptome and proteomics in the classical framework of human lifespan, considering that our samples' ages were not uniform along the age range and that the mRNA and protein 
datasets had different age windows, we interpolated 10 uniform points along the age range of each period, at the 0.25 -powered age scale $[24,99,100]$.

Spearman's rank correlation coefficient rho was calculated for each gene for development and aging periods, respectively, between mRNA and protein expression levels, based on interpolated points of spline curves (for details, see supplemental experimental procedures in Additional file 1).

\section{Equalization of expression and amplitude}

We simulated the distribution of the common part of the distributions of the developmental and aging intervals by average expression levels as well as by gene expression change amplitudes. Then, according to this distribution, we subsampled 1,000 times from agedependent genes, for each species. In each subsampling, we compared the median of correlation distributions between developmental and aging intervals by Wilcoxon test (for details, see supplemental experimental procedures in Additional file 1).

\section{Overlap of concordant/discordant gene groups between databases}

We calculated the overlap of concordant and discordant gene groups resulting from the original human time series dataset, with gene groups resulting independently from (1) a macaque time series dataset, (2) a different human RNA-seq time series dataset, (3) human mRNA and macaque protein expression, and (4) vice versa. We subsampled 1,000 times from all the age-dependent genes of two datasets. In each subsampling, we selected genes from each dataset of the same number as concordant/discordant gene groups and checked the number of overlapped genes, and compared real overlap amount with simulated overlap distribution.

\section{Clustering genes into groups}

We grouped concordant and discordant genes into four patterns of mRNA expression using k-means clustering. Before clustering, each gene was standardized to mean $=0$ and standard deviation $=1$. Because $\mathrm{k}$-means clustering is a heuristic algorithm, we repeated the procedure 1,000 times to determine the most frequent constellation and used this clustering in downstream analysis.

\section{Functional analysis}

We used the GO categories of biological process (BP), molecular function (MF) and cellular component (CC), together with KEGG pathway databases [28,29], for testing the functional enrichment of gene groups. To identify over-representation, we used GeneCoDis3, a non-redundant and modular enrichment analysis tool for functional annotation of gene sets [101] to investigate the putative functions of concordant and discordant gene groups in each of four patterns. Validated detected agedependent genes were taken as background, and hypergeometric test $P$-values were adjusted for multiple testing by the Benjamini-Hochberg correction.

\section{Posttranscriptional regulator RNA binding protein identification Extraction of exonic RNA binding protein binding sites}

All data sets from CLIP experiments (including HITSCLIP, PAR-CLIP and iCLIP) used in the analysis were collected from the published literature (Table S10 in Additional file 1).

The precise positional RBP target sites extracted from the CLIP data sets were mapped to gene locations by Ensembl gene annotation. We followed the cutoff used in the original literature of each CLIP experiment. Only binding sites passing the cutoff were used in the following analysis. To focus on post-transcriptional regulation corresponding to the mature mRNA, we excluded the binding sites located in intronic regions or in intron/ exon splicing junctions.

\section{Key regulator RNA binding protein identification}

To define a RBP as a key regulator of the disparity gene group of a pattern, we checked three conditions: (1) the regulator should target (has at least one validated binding site on the gene) a significantly higher percentage of discordant genes compared with concordant genes in the same pattern. The significance is indicated by $P<0.05$ in a binomial test after Bonferroni correction between percentages of targeted genes in concordant and discordant gene groups. (2) The regulator should have significantly more binding sites on the exon union of discordant genes compared with concordant genes in the same pattern. The binding site number enrichment is classified with $P<0.05$ of one-sided Wilcoxon test after Bonferroni correction between distributions of binding site number on genes in concordant and discordant gene groups. (3) After correction by the length of exon union for each gene, the regulator should have significantly higher density of binding sites in discordant genes compared with concordant genes in the same pattern. The significance is indicated by $P<0.05$ of one-sided Wilcoxon test after Bonferroni correction between distributions of binding site density of genes in concordant and discordant gene groups. One regulator is classified as a key regulator if it fulfils condition 1 and at least one of conditions 2 and 3.

\section{Posttranscriptional miRNA regulation estimation miRNA binding site extraction from in silico prediction} Human miRNA target prediction was based on miRNA target sites identified using Targetscan [102]. Furthermore, only target sites that were conserved in at least three out of 
four species (mouse, rat, dog and chicken) were classified as reliable conserved targets [103] (for details, see supplemental experimental procedures in Additional file 1).

\section{Enriched targeting miRNA identification}

To identify miRNA with enrichment of predicted targets in a discordant gene group in each of the four patterns, we compared miRNA binding site number with concordant genes in the same pattern. To avoid the influence of miRNA gene families, we restricted this comparison to a single test per unique miRNA seed. If a miRNA's predicted targets were enriched in a pattern after hypergeometric test $(P<0.05$ after Bonferroni correction), we considered that miRNA 'specific' to that discordant gene group. All the other miRNAs were considered 'non-specific' between the concordant and discordant gene groups in this pattern. These results were compared to chance distributions obtained using 1,000 permutations by randomized labeling of concordant/discordant genes within each pattern.

\section{Functional miRNA regulation estimation}

To estimate the negative effect of miRNAs on the translation of discordant genes in each pattern, we calculated the predicted miRNA-target distribution of the Spearman's rank correlation coefficients measured based on expression profiles of age-dependent miRNA and protein expression of their predicted target transcripts in aging interval.

The background chance distribution was generated by the same number of predicted miRNA-target pairs based on randomly chosen age-dependent miRNA and target genes within the discordant group of each pattern for 1,000 times.

We estimated the difference between predicted miRNAtarget distribution and background chance distribution by subtraction of the median of background chance distribution. A discordant group of one pattern was considered negatively regulated by miRNAs on the translation level if, and only if, significant excess of negative correlation $(P<0.05$, FDR $<5 \%$, Spearman's rank correlation) was observed. The significance is indicated by $95 \%$ confidence intervals of chance difference obtained in background chance distribution.

\section{Data availability}

All proteomics data have been deposited in the public PeptideAtlas database under accession number PASS00505.

\section{Additional files}

Additional file 1: Figures $\mathrm{S} 1$ to $\mathrm{S} 13$ and legends, supplemental Tables S1 to S8 and S10 and S11, and a complete and comprehensive list of experimental procedures and supplemental references.

Additional file 2: Table S9, which lists GO functional terms and KEGG pathways enriched in concordant and discordant genes.

\section{Abbreviations}

AGO: Argonaut; CLIP: crosslinking and immunoprecipitation; FDR: false discovery rate; GO: Gene Ontology; HITS-CLIP: high-throughput sequencing of RNA isolated by CLIP; iCLIP: individual-nucleotide resolution CLIP; KEGG: Kyoto Encyclopedia of Genes and Genomes; LC: liquid chromatography; miRNA: microRNA; MS/MS: tandem mass spectrometry; mTOR: mammalian target of rapamycin; NSAF: normalized spectral abundance factor; PAR-CLIP: photoactivatable-ribonucleoside-enhanced CLIP; PFC: prefrontal cortex; PI3K: phosphoinositide 3-kinase; RBP: RNA binding protein; RISC: RNA-induced silencing complex; RPKM: reads per kilobase per million of total mapped reads; SAF: spectral abundance factor; UTR: untranslated region.

\section{Competing interests}

The authors declare that they have no competing interests.

\section{Authors' contributions}

PK and RZ conceived and designed the experiments. NF and ZBN performed the experiments. YNW performed all data analyses, aside from the construction of the human-macaque consensus reference genome (GCX), computational pre-processing of miRNA deep sequencing and in silico prediction of miRNA binding sites $(\mathrm{HYH})$. PK and YNW wrote the manuscript. All authors read and approved the final manuscript.

\section{Acknowledgments}

We thank the NICHD Brain and Tissue Bank for Developmental Disorders, the Netherlands Brain Bank, and, in particular, HR Zielke and J Dai for providing the human samples; Suzhou Drug Safety Evaluation and Research Centre and C Lian, H Cai and X Zheng in particular for providing the macaque samples; J Jin for assistance; ZS He for assistance with statistical analysis; GL Banes for comments on the manuscript; and all other members of the Comparative Biology Group in Shanghai for helpful discussions and suggestions. This study was supported by the Chinese Academy of Sciences Strategic Priority Research Program (grant numbers XDB13010200), the Chinese Ministry of Science and Technology (2011CB910200), the National Natural Science Foundation of China (major research program, grant number 91331203; general program, grant number 31171232), the National One Thousand Foreign Experts Plan (grant number WQ20123100078), the Bureau of International Cooperation, Chinese Academy of Sciences (grant number GJHZ201313), and the Russian Science Foundation, grant 14-28-00234.

\section{Author details}

${ }^{1}$ CAS Key Laboratory of Computational Biology, CAS-MPG Partner Institute for Computational Biology, 320 Yue Yang Road, Shanghai 200031, China. ${ }^{2}$ University of the Chinese Academy of Sciences, Beijing 100039, China. ${ }^{3}$ Key Laboratory of Systems Biology, Institute of Biochemistry and Cell Biology, Shanghai Institutes for Biological Sciences, Chinese Academy of Sciences, 320 Yue Yang Road, Shanghai 200031, China. ${ }^{4}$ Max Planck Institute for Evolutionary Anthropology, Deutscher Platz 6, Leipzig 04103, Germany. ${ }^{5}$ Skoltech Center for Computational and Systems Biology, Skolkovo Institute for Science and Technology, Skolkovo 143025, Russia.

Received: 8 July 2014 Accepted: 9 February 2015

Published online: 22 February 2015

\section{References}

1. Yoo AS, Sun AX, Li L, Shcheglovitov A, Portmann T, Li Y, et al. MicroRNA-mediated conversion of human fibroblasts to neurons. Nature. 2011;476:228-31.

2. van Kouwenhove M, Kedde M, Agami R. MicroRNA regulation by RNA-binding proteins and its implications for cancer. Nat Rev Cancer. 2011;11:644-56

3. Siegel G, Saba R, Schratt G. microRNAs in neurons: manifold regulatory roles at the synapse. Curr Opin Genet Dev. 2011;21:491-7.

4. Janga SC, Vallabhaneni S. MicroRNAs as post-transcriptional machines and their interplay with cellular networks. Adv Exp Med Biol. 2011;722:59-74.

5. Glisovic T, Bachorik JL, Yong J, Dreyfuss G. RNA-binding proteins and post-transcriptional gene regulation. FEBS Lett. 2008;582:1977-86.

6. Ray D, Kazan H, Cook KB, Weirauch MT, Najafabadi HS, Li X, et al. A compendium of RNA-binding motifs for decoding gene regulation. Nature. 2013;499:172-7. 
7. Vindry C, Ngoc LV, Kruys V, Gueydan C. RNA-binding protein-mediated posttranscriptional controls of gene expression: Integration of molecular mechanisms at the 3' end of mRNAs? Biochem Pharmacol. 2014;89:431-40.

8. Cook KB, Kazan H, Zuberi K, Morris Q, Hughes TR. RBPDB: a database of RNA-binding specificities. Nucleic Acids Res. 2011;39:D301-8.

9. Licatalosi DD, Mele A, Fak JJ, Ule J, Kayikci M, Chi SW, et al. HITS-CLIP yields genome-wide insights into brain alternative RNA processing. Nature. 2008;456:464-9.

10. Hafner M, Landthaler M, Burger L, Khorshid M, Hausser J, Berninger $P$, et al. PAR-CliP--a method to identify transcriptome-wide the binding sites of RNA binding proteins. J Vis Exp. 2010. doi: 10.3791/2034.

11. Konig J, Zarnack K, Rot G, Curk T, Kayikci M, Zupan B, et al. iCLIP reveals the function of hnRNP particles in splicing at individual nucleotide resolution. Nat Struct Mol Biol. 2010;17:909-15.

12. Kozomara A, Griffiths-Jones S. miRBase: integrating microRNA annotation and deep-sequencing data. Nucleic Acids Res. 2011;39:D152-7.

13. Meister G. Argonaute proteins: functional insights and emerging roles. Nat Rev Genet. 2013;14:447-59.

14. Pasquinelli AE. MicroRNAs and their targets: recognition, regulation and an emerging reciprocal relationship. Nat Rev Genet. 2012;13:271-82.

15. Fabian MR, Sonenberg N, Filipowicz W. Regulation of mRNA translation and stability by microRNAs. Annu Rev Biochem. 2010;79:351-79.

16. Valdes AM, Glass D, Spector TD. Omics technologies and the study of human ageing. Nat Rev Genet. 2013;14:601-7.

17. Sayed D, Abdellatif M. MicroRNAs in development and disease. Physiol Rev. 2011;91:827-87.

18. Steffen KK, MacKay VL, Kerr EO, Tsuchiya M, Hu D, Fox LA, et al. Yeast life span extension by depletion of 60s ribosomal subunits is mediated by Gcn4. Cell. 2008;133:292-302.

19. Hansen M, Taubert S, Crawford D, Libina N, Lee SJ, Kenyon C. Lifespan extension by conditions that inhibit translation in Caenorhabditis elegans. Aging Cell. 2007;6:95-110.

20. Bjedov I, Toivonen JM, Kerr F, Slack C, Jacobson J, Foley A, et al. Mechanisms of life span extension by rapamycin in the fruit fly Drosophila melanogaster. Cell Metab. 2010;11:35-46.

21. Sharp ZD, Bartke A. Evidence for down-regulation of phosphoinositide 3-kinase/Akt/mammalian target of rapamycin (PI3K/Akt/mTOR)-dependent translation regulatory signaling pathways in Ames dwarf mice. J Gerontol A Biol Sci Med Sci. 2005;60:293-300

22. Mazin P, Xiong J, Liu X, Yan Z, Zhang X, Li M, et al. Widespread splicing changes in human brain development and aging. Mol Syst Biol. 2013;9:633.

23. Lu T, Pan Y, Kao SY, Li C, Kohane I, Chan J, et al. Gene regulation and DNA damage in the ageing human brain. Nature. 2004;429:883-91.

24. Rodwell GE, Sonu R, Zahn JM, Lund J, Wilhelmy J, Wang L, et al. A transcriptional profile of aging in the human kidney. PLoS Biol. 2004;2:e427.

25. de Magalhaes JP, Costa J. A database of vertebrate longevity records and their relation to other life-history traits. J Evol Biol. 2009:22:1770-4.

26. Walker R, Gurven M, Hill K, Migliano A, Chagnon N, De Souza R, et al. Growth rates and life histories in twenty-two small-scale societies. Am J Hum Biol. 2006;18:295-311.

27. He Z, Bammann H, Han D, Xie G, Khaitovich P. Conserved expression of lincRNA during human and macaque prefrontal cortex development and maturation. RNA. 2014:20:1103-11.

28. Kanehisa M, Goto S, Sato Y, Kawashima M, Furumichi M, Tanabe M. Data, information, knowledge and principle: back to metabolism in KEGG. Nucleic Acids Res. 2014;42:D199-205.

29. Reference Genome Group of the Gene Ontology C. The Gene Ontology's Reference Genome Project: a unified framework for functional annotation across species. PLoS Comput Biol. 2009;5:e1000431

30. Kishore S, Jaskiewicz L, Burger L, Hausser J, Khorshid M, Zavolan M. A quantitative analysis of CLIP methods for identifying binding sites of RNA-binding proteins. Nat Methods. 2011;8:559-64.

31. Hoell Jl, Larsson E, Runge S, Nusbaum JD, Duggimpudi S, Farazi TA, et al RNA targets of wild-type and mutant FET family proteins. Nat Struct Mol Biol. 2011;18:1428-31.

32. Ascano Jr M, Mukherjee N, Bandaru P, Miller JB, Nusbaum JD, Corcoran DL, et al. FMRP targets distinct mRNA sequence elements to regulate protein expression. Nature. 2012;492:382-6.

33. Hafner M, Landthaler M, Burger L, Khorshid M, Hausser J, Berninger $P$, et al. Transcriptome-wide identification of RNA-binding protein and microRNA target sites by PAR-CLIP. Cell. 2010;141:129-41.
34. Sievers C, Schlumpf T, Sawarkar R, Comoglio F, Paro R. Mixture models and wavelet transforms reveal high confidence RNA-protein interaction sites in MOV10 PAR-CLIP data. Nucleic Acids Res. 2012;40:e160.

35. Sanford JR, Wang X, Mort M, Vanduyn N, Cooper DN, Mooney SD, et al. Splicing factor SFRS1 recognizes a functionally diverse landscape of RNA transcripts. Genome Res. 2009;19:381-94.

36. Tollervey JR, Curk T, Rogelj B, Briese M, Cereda M, Kayikci M, et al. Characterizing the RNA targets and position-dependent splicing regulation by TDP-43. Nat Neurosci. 2011;14:452-8.

37. Wang Z, Kayikci M, Briese M, Zarnack K, Luscombe NM, Rot G, et al. iCLIP predicts the dual splicing effects of TIA-RNA interactions. PLoS Biol. 2010;8:e1000530.

38. Sanford JR, Gray NK, Beckmann K, Caceres JF. A novel role for shuttling SR proteins in mRNA translation. Genes Dev. 2004;18:755-68.

39. Mazan-Mamczarz K, Lal A, Martindale JL, Kawai T, Gorospe M. Translational repression by RNA-binding protein TIAR. Mol Cell Biol. 2006;26:2716-27.

40. Somel M, Guo S, Fu N, Yan Z, Hu HY, Xu Y, et al. MicroRNA, mRNA, and protein expression link development and aging in human and macaque brain. Genome Res. 2010;20:1207-18.

41. Wang X. miRDB: a microRNA target prediction and functional annotation database with a wiki interface. RNA. 2008;14:1012-7.

42. Gennarino VA, D'Angelo G, Dharmalingam G, Fernandez S, Russolillo G, Sanges $\mathrm{R}$, et al. Identification of microRNA-regulated gene networks by expression analysis of target genes. Genome Res. 2012;22:1163-72.

43. Damgaard CK, Lykke-Andersen J. Translational coregulation of 5TOP mRNAs by TIA-1 and TIAR. Genes Dev. 2011;25:2057-68.

44. ladevaia V, Caldarola S, Tino E, Amaldi F, Loreni F. All translation elongation factors and the $\mathrm{e}, \mathrm{f}$, and $\mathrm{h}$ subunits of translation initiation factor 3 are encoded by 5'-terminal oligopyrimidine (TOP) mRNAs. RNA. 2008;14:1730-6.

45. Johnson SC, Rabinovitch PS, Kaeberlein M. mTOR is a key modulator of ageing and age-related disease. Nature. 2013;493:338-45.

46. Bonawitz ND, Chatenay-Lapointe M, Pan Y, Shadel GS. Reduced TOR signaling extends chronological life span via increased respiration and upregulation of mitochondrial gene expression. Cell Metab. 2007:5:265-77.

47. Pan Y, Schroeder EA, Ocampo A, Barrientos A, Shadel GS. Regulation of yeast chronological life span by TORC1 via adaptive mitochondrial ROS signaling. Cell Metab. 2011;13:668-78.

48. Polak P, Cybulski N, Feige JN, Auwerx J, Ruegg MA, Hall MN. Adiposespecific knockout of raptor results in lean mice with enhanced mitochondrial respiration. Cell Metab. 2008;8:399-410.

49. Hassan B, Akcakanat A, Holder AM, Meric-Bernstam F. Targeting the PI3-kinase/Akt/mTOR signaling pathway. Surg Oncol Clin N Am. 2013;22:641-64

50. O' Neill C. PI3-kinase/Akt/mTOR signaling: impaired on/off switches in aging cognitive decline and Alzheimer's disease. Exp Gerontol. 2013:48:647-53.

51. Ma XM, Blenis J. Molecular mechanisms of mTOR-mediated translational control. Nat Rev Mol Cell Biol. 2009:10:307-18.

52. Holz MK, Ballif BA, Gygi SP, Blenis J. mTOR and S6K1 mediate assembly of the translation preinitiation complex through dynamic protein interchange and ordered phosphorylation events. Cell. 2005;123:569-80.

53. Johnson TE. Caenorhabditis elegans 2007: the premier model for the study of aging. Exp Gerontol. 2008;43:1-4

54. Kenyon CJ. The genetics of ageing. Nature. 2010:464:504-12.

55. Maiese K, Chong ZZ, Shang YC, Wang S. mTOR: on target for novel therapeutic strategies in the nervous system. Trends Mol Med. 2013:19:51-60.

56. Troca-Marin JA, Alves-Sampaio A, Montesinos ML. An increase in basal BDNF provokes hyperactivation of the Akt-mammalian target of rapamycin pathway and deregulation of local dendritic translation in a mouse model of Down's syndrome. J Neurosci. 2011:31:9445-55.

57. Kadish I, Thibault O, Blalock EM, Chen KC, Gant JC, Porter NM, et al. Hippocampal and cognitive aging across the lifespan: a bioenergetic shift precedes and increased cholesterol trafficking parallels memory impairment. J Neurosci. 2009;29:1805-16.

58. Yang F, Chu X, Yin M, Liu X, Yuan H, Niu Y, et al. mTOR and autophagy in normal brain aging and caloric restriction ameliorating age-related cognition deficits. Behav Brain Res. 2014;264:82-90.

59. Thoreen CC, Chantranupong L, Keys HR, Wang T, Gray NS, Sabatini DM. A unifying model for mTORC1-mediated regulation of mRNA translation. Nature. 2012;485:109-13.

60. Yang Q, Guan KL. Expanding mTOR signaling. Cell Res. 2007;17:666-81. 
61. Mamane $Y$, Petroulakis E, LeBacquer O, Sonenberg N. mTOR, translation initiation and cancer. Oncogene. 2006;25:6416-22.

62. Laplante M, Sabatini DM. mTOR signaling at a glance. J Cell Sci. 2009:122:3589-94

63. Fabrizio P, Pozza F, Pletcher SD, Gendron CM, Longo VD. Regulation of longevity and stress resistance by Sch9 in yeast. Science. 2001;292:288-90.

64. Pan KZ, Palter JE, Rogers AN, Olsen A, Chen D, Lithgow GJ, et al. Inhibition of mRNA translation extends lifespan in Caenorhabditis elegans. Aging Cell. 2007;6:111-9.

65. Kapahi P, Zid BM, Harper T, Koslover D, Sapin V, Benzer S. Regulation of lifespan in Drosophila by modulation of genes in the TOR signaling pathway. Curr Biol. 2004;14:885-90.

66. Selman C, Tullet JM, Wieser D, Irvine E, Lingard SJ, Choudhury Al, et al. Ribosomal protein $\mathrm{S} 6$ kinase 1 signaling regulates mammalian life span. Science. 2009;326:140-4.

67. Ayyadevara S, Alla R, Thaden JJ, Shmookler Reis RJ. Remarkable longevity and stress resistance of nematode PI3K-null mutants. Aging Cell. 2008;7:13-22.

68. Smith ED, Tsuchiya M, Fox LA, Dang N, Hu D, Kerr EO, et al. Quantitative evidence for conserved longevity pathways between divergent eukaryotic species. Genome Res. 2008:18:564-70.

69. Kaeberlein M, Kennedy BK. Protein translation, 2007. Aging Cell. 2007:6:731-4.

70. Blagosklonny MV. Aging and immortality: quasi-programmed senescence and its pharmacologic inhibition. Cell Cycle. 2006;5:2087-102.

71. Polivka Jr J, Janku F. Molecular targets for cancer therapy in the PI3K/AKT/ mTOR pathway. Pharmacol Ther. 2014;142:164-75.

72. Tong X, Pelling JC. Targeting the PI3K/Akt/mTOR axis by apigenin for cancer prevention. Anticancer Agents Med Chem. 2013;13:971-8.

73. Schieke SM, Phillips D, McCoy Jr JP, Aponte AM, Shen RF, Balaban RS, et al. The mammalian target of rapamycin (mTOR) pathway regulates mitochondrial oxygen consumption and oxidative capacity. J Biol Chem. 2006;281:27643-52.

74. Ramanathan A, Schreiber SL. Direct control of mitochondrial function by mTOR. Proc Natl Acad Sci U S A. 2009;106:22229-32.

75. Lee HC, Wei YH. Mitochondria and aging. Adv Exp Med Biol. 2012:942:311-27.

76. Wei YH, Wu SB, Ma YS, Lee HC. Respiratory function decline and DNA mutation in mitochondria, oxidative stress and altered gene expression during aging. Chang Gung Med J. 2009;32:113-32.

77. Caballero A, Ugidos A, Liu B, Oling D, Kvint K, Hao X, et al. Absence of mitochondrial translation control proteins extends life span by activating sirtuin-dependent silencing. Mol Cell. 2011:42:390-400.

78. Bahadorani S, Cho J, Lo T, Contreras H, Lawal HO, Krantz DE, et al. Neuronal expression of a single-subunit yeast NADH-ubiquinone oxidoreductase (Ndi1) extends Drosophila lifespan. Aging Cell. 2010;9:191-202.

79. Zid BM, Rogers AN, Katewa SD, Vargas MA, Kolipinski MC, Lu TA, et al. 4E-BP extends lifespan upon dietary restriction by enhancing mitochondrial activity in Drosophila. Cell. 2009;139:149-60

80. Lau P, Bossers K, Janky R, Salta E, Frigerio CS, Barbash S, et al. Alteration of the microRNA network during the progression of Alzheimer's disease. EMBO Mol Med. 2013:5:1613-34.

81. Wong HK, Veremeyko T, Patel N, Lemere CA, Walsh DM, Esau C, et al. De-repression of FOXO3a death axis by microRNA-132 and -212 causes neuronal apoptosis in Alzheimer's disease. Hum Mol Genet. 2013:22:3077-92.

82. Wanet A, Tacheny A, Arnould T, Renard P. miR-212/132 expression and functions: within and beyond the neuronal compartment. Nucleic Acids Res. 2012:40:4742-53.

83. Shukla U, Tumma N, Gratsch T, Dombkowski A, Novak RF. Insights into insulin-mediated regulation of CYP2E1: miR-132/-212 targeting of CYP2E and role of phosphatidylinositol 3-kinase, Akt (protein kinase B), mammalian target of rapamycin signaling in regulating miR-132/-212 and miR-122/-181a expression in primary cultured rat hepatocytes. Drug Metab Dispos. 2013:41:1769-77.

84. Fu X, Fu N, Guo S, Yan Z, Xu Y, Hu H, et al. Estimating accuracy of RNA-Seq and microarrays with proteomics. BMC Genomics. 2009:10:161.

85. Schwartz S, Kent WJ, Smit A, Zhang Z, Baertsch R, Hardison RC, et al. Human-mouse alignments with BLASTZ. Genome Res. 2003;13:103-7.

86. Wheeler DL, Barrett T, Benson DA, Bryant SH, Canese K, Chetvernin V, et al. Database resources of the National Center for Biotechnology Information. Nucleic Acids Res. 2008;36:D13-21.
87. Dobin A, Davis CA, Schlesinger F, Drenkow J, Zaleski C, Jha S, et al. STAR: ultrafast universal RNA-seq aligner. Bioinformatics. 2013;29:15-21.

88. Li H, Handsaker B, Wysoker A, Fennell T, Ruan J, Homer N, et al. The Sequence Alignment/Map format and SAMtools. Bioinformatics. 2009;25:2078-9.

89. Harrow J, Denoeud F, Frankish A, Reymond A, Chen CK, Chrast J, et al. GENCODE: producing a reference annotation for ENCODE. Genome Biol. 2006;7:S4. 1-9.

90. Harrow J, Frankish A, Gonzalez JM, Tapanari E, Diekhans M, Kokocinski F, et al. GENCODE: the reference human genome annotation for The ENCODE Project. Genome Res. 2012:22:1760-74.

91. UniProt C. Activities at the Universal Protein Resource (UniProt). Nucleic Acids Res. 2014:42:D191-8.

92. Granholm V, Kim S, Navarro JC, Sjolund E, Smith RD, Kall L. Fast and accurate database searches with MS-GF + Percolator. J Proteome Res. 2014;13:890-7.

93. Zhu W, Smith JW, Huang CM. Mass spectrometry-based label-free quantitative proteomics. J Biomed Biotechnol. 2010;2010:840518.

94. Barrett T, Wilhite SE, Ledoux P, Evangelista C, Kim IF, Tomashevsky M, et al. NCBI GEO: archive for functional genomics data sets - update. Nucleic Acids Res. 2013:41:D991-5.

95. Hu HY, Yan Z, Xu Y, Hu H, Menzel C, Zhou YH, et al. Sequence features associated with microRNA strand selection in humans and flies. BMC Genomics. 2009:10:413.

96. Langmead B, Trapnell C, Pop M, Salzberg SL. Ultrafast and memory-efficient alignment of short DNA sequences to the human genome. Genome Biol. 2009;10:R25

97. Griffiths-Jones S, Grocock RJ, van Dongen S, Bateman A, Enright AJ. miRBase: microRNA sequences, targets and gene nomenclature. Nucleic Acids Res. 2006:34:D140-4.

98. Somel M, Franz H, Yan Z, Lorenc A, Guo S, Giger T, et al. Transcriptional neoteny in the human brain. Proc Natl Acad Sci U S A. 2009;106:5743-8.

99. Clancy B, Darlington RB, Finlay BL. Translating developmental time across mammalian species. Neuroscience. 2001;105:7-17.

100. Erraji-Benchekroun L, Underwood MD, Arango V, Galfalvy H, Pavlidis P, Smyrniotopoulos $P$, et al. Molecular aging in human prefrontal cortex is selective and continuous throughout adult life. Biol Psychiatry. 2005;57:549-58

101. Tabas-Madrid D, Nogales-Cadenas R, Pascual-Montano A. GeneCodis3: a non-redundant and modular enrichment analysis tool for functiona genomics. Nucleic Acids Res. 2012;40:W478-83.

102. Lewis BP, Burge CB, Bartel DP. Conserved seed pairing, often flanked by adenosines, indicates that thousands of human genes are microRNA targets. Cell. 2005:120:15-20.

103. Edgar RC. MUSCLE: multiple sequence alignment with high accuracy and high throughput. Nucleic Acids Res. 2004;32:1792-7.

\section{Submit your next manuscript to BioMed Central and take full advantage of:}

- Convenient online submission

- Thorough peer review

- No space constraints or color figure charges

- Immediate publication on acceptance

- Inclusion in PubMed, CAS, Scopus and Google Scholar

- Research which is freely available for redistribution 